\title{
Garde ta salive pour embrasser les garçons
}

\author{
Isabelle Décarie
}

\begin{abstract}
Does a woman experience misogyny more acutely during her adolescence, while learning of the world and of sexuality, at a time when her vulnerability is at its peak? Through accounts of female coming of age, the present text explores these questions from the angle of experience and tries to establish recent cultural and social phenomena which revived the debate on misogyny.
\end{abstract}

Mon cahier des charges est enflé de doléances et au chapitre de la misogynie, on y trouve des scènes, des épisodes, des phrases qui me redisent encore aujourd'hui combien grandir quand on est une fille revient souvent à jouer la potiche: la misogynie existe, elle est insidieuse et pernicicuse, elle agit en tout temps et en tout lieu, personne ne peut la combattre, elle est un mal nécessaire, oublie-la. Sois belle et tais-toi.

Je travaillais à temps partiel dans un bureau de typographie pour payer mes études. L'espace était divisé en deux parties: devant, à la réception, se trouvaient les femmes, derrière, à la production, les hommes. Je travaillais avec N., la belle Italienne haïtienne qui habitait à Laval et dont $\mathrm{j}$ 'aimais tant le cynisme; avec A. qui était si innocente et dont $\mathrm{j}$ 'avais peur d'attraper le mode de vie (mariée jeune, cours par correspondance, maison semi-détachée à Saint-Constant) et S., l'Anglo, la chef de service, apathique, lente, toujours en retard, flemmarde. Malgré leurs défauts, elles étaient mes alliées, nous représentions le front, la devanture. Nous complotions tant que nous pouvions contre le propriétaire, B., un minable boutonneux. J'avais pourtant été heureuse d'être employée par lui car je pensais trouver chez cet homosexuel un terrain neutre où je pourrais être une fille sans en pâtir. Un soir, alors que j'étais la dernière à la réception (dernière embauchée, dernière à partir), B. est venu parler au dernier client dans la salle d'attente. Le bureau avait été récemment rénové et le client n'avait encore rien vu. Il complimentait $B$. pour les nouveaux ajouts. Ce dernier lui dit alors en me désignant derrière le comptoir: «Mais ce n'est pas tout, nous avons aussi de nouveaux accessoires». Rires sarcastiques des deux, rictus estomaqué pour moi. J'avais été reléguée au rayon tapisserie. Même les gais s'y mettaient. C'était la même année que la Poly.

J'avais des réflexes de fille qui se sentait toujours à part, rejetée, jamais au bon endroit au bon moment, en décalage constant. Je cultivais une posture de potiche, de martyre, de victime, de femme détestée. Et c'est pourquoi je passais mon temps à faire des calculs du ratio homme / femme à partir de listes de tout acabit (gagnants d'un concours radio, auteurs publiés chez un éditeur, professeurs dans un département, membres d'un conseil d'administration, délégués politiques, 
médecins dans un cabinet). Ma savante comptabilité confortait mon attitude: la majorité était trop souvent masculine. Je râlais constamment, je signalais à tous et toutes leurs propos misogynes, je fulminais, j'enrageais, je ne laissais rien passer. J'écrivais aux journaux, je criais à la télévision, je déchirais les livres. Un jour, j'ai fait remarquer à un journaliste que la liste des cent chef-d'œuvres de la littérature mondiale qu'il avait établie ne comportait que trois femmes. Excédé, d'un regard sans appel, il a rétorqué que ses choix reflétaient simplement, tout simplement, la réalité. J'avais découpé une publicité pour un four où l'on voyait une femme en tablier, alanguie, belle, en talons hauts, servir un homme. Le four portait un nom évocateur: Caprice. Je brandissais la page découpée et je déclarais mon indignation à qui voulait bien l'entendre. Je militais surtout dans le salon de mes parents, convaincue que misogynie bien ordonnée commence par chez soi. Un ami de mon père m'a avoué, en me tirant durement par la manche et en s'approchant très près de ma bouche tordue par la honte et la colère, que si je continuais comme ça, je ne trouverais jamais de mari. Ma copine A.-I. qui s'est fait mettre un doigt dans la culotte par un inconnu sur le chemin de la maison m'a dit que j'étais tristement féministe de paraître outrée d'un tel phénomène: c'était bien naturel qu'on se serve d'elle, les hommes avaient des besoins à assouvir même si c'était sous une pluie d'injures, la tête éclatée contre un poteau de téléphone. Quand ma mère m'a dit que je ne serais sans doute pas féministe si j'étais grande et blonde, quand un travesti m'a avoué sa haine pour les femmes, j'ai rangé mes munitions. Je me suis tue. Est-ce que je faisais des histoires pour rien? Des histoires comme des caprices de jeune fille? Grandir, c'est ça ma fille. C'est comprendre l'inutilité de ce que tu dis, de ce que tu es. Garde ta salive pour embrasser les garçons.

Ces récits d'adolescence font partie du passé. Ces scènes ont été vécues comme des moments de misogynie. Mais aujourd'hui, ils me rappellent surtout la difficile mutation du corps et du désir à un âge ingrat, l'apprentissage de la différence des sexes par la violence, la menace du poing, la tempe fêlée, la manche tirée, le poignet rougi. Une différence qui chaque fois creuse un écart comme une fosse. Depuis que je me suis roulée de honte d'être de mon sexe en regardant tant de filles mourir à la télé, je ne pense plus à la misogynie.

Je ne pense plus à la misogynie parce que je ne sais plus comment la penser.

Une fois qu'on a compris que «tenir la femme en respect» (Sarah Kofman), c'est aussi faire preuve de misogynie, une fois qu'on a renversé les oppositions en disant que la misogynie, somme toute, n'est que la manifestation d'une peur ancestrale du sexe féminin ouvert, profond, caverneux, tranchant et castrateur (Freud), une fois qu'on a rappelé que le supplément féminin de Lacan (qu'on avait, dans un premier temps, compris comme une plus-value) n'est qu'une autre façon de mettre «une femme» à l'écart (la femme n'existant pas, on s'en souvient), une fois qu'on a bien perçu que certaines positions féministes ne font que renverser les discours en empruntant aux misogynes leurs arguments et leurs armes (Naomi Wolf), que les mesures pour corriger certaines absurdités linguistiques ou absences politiques (féminisation de la langue au Québec, parité en France souvent considérée «humiliante» par certaines femmes ) changent peu de 
choses à l'affaire, que le discours sur la misogynie aujourd'hui est surtout relayé par les femmes et dirigé contre elles-mêmes (le film Mean Girls), alors, comment, finalement, penser la misogynie? Est-ce que ce terme (et tout ce qu'il recouvre) nous dit encore quelque chose? Souvent relié au féminisme d'un autre temps, aurait-il mal vieilli? Ou est-ce moi qui ai vieilli?

Peut-être n'est-ce pas tant le mot lui-même que les luttes constantes pour «l'égalité» qui semblent datées. C'est aussi ce que note comme Camille Laurens dans Le Grain des mots. À l'entrée «Homme / Femme», elle raconte qu'il y a quelques années, elle enseignait à des étudiants marocains en classes préparatoires: «le thème au programme était: L'Histoire, et $\mathrm{j}$ 'avais posé le très classique sujet de dissertation suivant: "Les hommes font-ils l'histoire?" Parmi des devoirs plus ou moins argumentés, se trouvait cette réponse sans appel: "Les hommes, oui, mais pas les femmes. Les femmes, elles, font des histoires"》». Camille Laurens réfléchit sur le mot «Homme» qui désigne à la fois l'espèce humaine et «l'individu mâle». L'iniquité de la langue est depuis toujours matière à débat puisqu'un homme peut dire «c'est ma femme» mais une femme ne peut pas dire sans connotation péjorative «c'est mon homme». Laurens termine son petit texte en disant ceci: «Dépassons pourtant ces vieux, très vieux conflits et observons que, in fine, les deux mots [homme et femme ] sont assez semblables. Ce qui les rapproche surtout, au-delà des divergences initiales, ce sont les deux $\mathrm{m}$ qui en forment le cœur. Voici donc la bonne nouvelle (le e reste muet de ravissement): homme, femme, les deux s'aiment».

Les deux m se lovent donc mais naïvement et ils se collent dans l'amour qu'ils ont l'un pour l'autre. On voudrait pourtant croire que les deux s'aiment du même amour. In fine, un ravissement c'est aussi un rapt. Si les femmes font l'Histoire, elles font aussi des histoires, et doivent continuer à en faire; elles ne peuvent pas se taire, elles doivent donner du fil à retordre, faire des ennuis, générer des disputes. Laurens ne dit rien (ou elle dit tout en voulant évacuer le débat, en le contournant bien vite) du fait que ce jeune garçon soit marocain, sachant pertinemment qu'en France il s'agit-là d'un débat délicat, rendu d'autant plus sensible depuis «l'affaire du voile». Au Québec, dans les tribunes publiques et dans les journaux, on a souvent applaudi la loi française empêchant les filles de porter le voile à l'école parce que cette loi réaffirmait l'importance de la laïcité (une laïcité qui n'est qu'un prétexte par ailleurs), mais surtout parce qu'elle permettait ainsi de mettre un terme à une pratique misogyne, au moins pendant les heures où les étudiantes étaient en classe. Sommes-nous assez naïves pour penser que cette loi va changer quoi que ce soit dans les foyers? que ce geste étatique est une partie gagnée pour une certaine «cause des femmes»? que nous pouvons nous interposer entre nos idéaux soi-disant démocratiques et les dogmes d'une religion? L'hypocrisie fait parfois plus de torts que la misogynie et cette dernière n'est pas le seul apanage des garçons marocains.

On peut penser que la misogynie n'existe plus parce que les femmes ont maintenant le choix, le pouvoir de tout décider. La vie serait devenue un grand buffet chinois où l'on pourrait sélectionner toutes les combinaisons possibles: enfants sans homme, carrière avec un homme mais sans enfants, un homme ou 
une femme avec enfants et carrière, etc. Il me semble que les choses ne sont pas aussi simples, tout particulièrement parce que nous ne sommes pas tous d'accord sur ce que choisir veut dire, parce que dans l'esprit de certains et de certaines, il y a des choix plus valables que d'autres. Le récent remake du film Stepford Wives a été l'occasion de nombreux débats dans les médias. Ce film raconte l'histoire d'une petite ville américaine dans laquelle les maris transforment leurs femmes en robots ménagers. Dans la version de 1975, Stepford Wives était un film d'horreur. Aujourd'hui, le script a été réécrit de manière à en faire une comédie. Certains critiques ont interprété la version contemporaine comme une machine machiste et misogyne, un véhicule promotionnel pour les hommes roses de la période postféministe en mal d'identité. D'autres ont tout simplement pensé que ce film permettait aux hommes et aux femmes de rire ensemble d'un fait de société qui ne les concernait plus. Ce qui semble le plus frappant ici, ce n'est pas tant le fait que les femmes mises en scène arborent des seins en silicone et des tabliers acidulés, mais bien que ce film soit devenu une comédie. Si la matière même du film prête le flanc à la critique, au moins, dans la première version, le sentiment d'inquiétante étrangeté dégagé par ces fembots était pour le moins menaçant: il laissait percevoir l'ambiguïté du phantasme de transformation. À qui appartenait vraiment ce phantasme? Était-ce un désir uniquement masculin? La version comique du film laisse penser justement que rester à la maison et élever une famille est encore un stéréotype misogyne dont il faut se moquer, dont il faut rire à gorge déployée. Le rire a bon dos. Certes, on pourra arguer que ce sont les hommes qui poussent les femmes dans ce film à tenir maison: elles n'ont pas fait ce choix. Ce film nous rappelle encore que les tâches domestiques et le soin des enfants restent des activités méprisées. Daphne Merkin dans sa propre critique du film est consternée parce que les choses n'ont pas changé depuis les années soixante: sa fille Zoë qui a 14 ans hésite entre devenir pédoneurochirurgienne et mère au foyer pour élever à temps plein trois enfants ${ }^{\mathrm{ii}}$. Cette hésitation pour Merkin reflète la pérennité de la misogynie. Le seul fait que sa fille manifeste l'envie d'élever ses enfants en restant à la maison illustre, selon elle, ce qu'Anna Fels dans son livre Necessary Dreams a remarqué (elle aussi avec consternation) après avoir interviewé de nombreuses jeunes femmes, à savoir que le mot «ambition» leur fait peur alors qu'elles veulent plus que jamais faire carrière. Pour les femmes nées dans les années cinquante, l'ambition était la clé de voûte de leur intégrité: elles se devaient de faire carrière afin de démontrer que le féminisme avait porté fruits. Une femme sans ambition ne pouvait que comploter avec l'ennemi.

Il me semble pourtant que c'est Daphne Merkin qui continue de penser en des termes misogynes. Sa fille, elle, a bien compris ce que la génération de sa mère a complètement exclu: la famille peut aussi être un choix valide, équivalent à la pédoneurochirurgie. Ce qui est triste pourtant, c'est qu'il faille élever non pas un, ni deux mais bien trois enfants dans l'esprit de Zoë pour que ce choix soit valorisé. Rester à la maison pour en élever un seul serait sans doute bien paresseux. Le buffet chinois aurait ses limites: tous les plats ne se valent pas. 
Peut-être penserons-nous un jour qu'il est rétrograde et misogyne de considérer l'indépendance financière et l'ambition professionnelle des femmes comme les éléments clés de leur liberté. Car dire cela, c'est répéter encore qu'elles ne sont pas libres de choisir de n'être rien ou d'être tout simplement; c'est continuer de transmettre l'idée qu'elles doivent «se réaliser», «s'assumer» pour pouvoir espérer tenir tête ou être à la hauteur de leurs frères. De la même façon, il faut sans doute espérer abolir le 8 mars, fête de la femme, pouvoir haïr les femmes sans être inculpé. Un tel paradoxe serait la preuve de la fin de la misogynie. Plus de ghettoïsation des sentiments, plus de rectitude politique, plus de censure. Jusqu'où pourra-t-on aller? Jusqu'où faut-il aller?

Je ne veux plus être de celles qui montent la garde, de celles qui font la vigie à la devanture du féminisme. Je ne veux plus être le chien de garde hargneux qui montre les dents dès qu'une parole menace ce que je suis. Mais si j'ai une fille un jour, que vais-je lui dire? Je vais sans doute lui mentir. Sois libre de faire des potiches, la potiche, des histoires ou l'Histoire, sois belle et fais le bon choix.

\section{Notes}

i Voir l'opinion d'Élisabeth Roudinesco à ce sujet dans Jacques Derrida, Élisabeth Roudinesco, De quoi demain..., Paris: Fayard / Galilée, 2001, p. 47.

ii Daphne Merkin, "The Machines Men Still Want? ", New York Times Magazine, 13 juin 2004, p. 15-16. 